\title{
Three Orthogonal Chromophores Operating Independently within the Same Molecule*
}

\author{
Heinz Langhals*, ${ }^{a}$ Maximilian Rauscher, ${ }^{a}$ Jörg Strübe, ${ }^{b}$ and Dietmar Kuck ${ }^{* b}$ \\ aDepartment of Chemistry, LMU University of Munich, Butenandtstraße 13, 81377 Munich, \\ Germany, and \\ ${ }^{b}$ Department of Chemistry, Bielefeld University, Universitätsstraße 25, 33651 Bielefeld, \\ Germany
}

\section{Supporting Information}

\section{List of Contents}

1. ${ }^{1} \mathrm{H}$ and ${ }^{13} \mathrm{C}$ NMR spectra

Page $S 2$

1.1. ${ }^{1} \mathrm{H}$ NMR spectrum of compound $8 \mathbf{a}$ (enriched mixture)

Page $S 2$

1.2. ${ }^{13} \mathrm{C}$ NMR spectrum of compound $\mathbf{8 a}$ (enriched mixture)

Page $S 3$

1.3. ${ }^{1} \mathrm{H}$ NMR spectrum of compound $9 \mathrm{a}$

Page $S 4$

1.4. ${ }^{13} \mathrm{C}$ NMR spectrum of compound 9a

Page $S 5$

1.5. ${ }^{1} \mathrm{H}$ NMR spectrum of the mixture of $8 \mathbf{b} / 9 \mathbf{b}$

Page $S 6$

1.6. ${ }^{13} \mathrm{C}$ NMR spectrum of the mixture of $8 \mathbf{b} / \mathbf{9 b}$

Page $S 7$

2. Mass spectrometric data of compound $\mathbf{8 a}$

Page S 8

2.1 MALDI-TOF mass spectrum of $\mathbf{8 a}$ at low resolution

Page S 8

2.2 MALDI-FT-ICR accurate mass measurement of $\mathbf{8 a}$ at high resolution

Page S 9

3. UV/Vis spectra

Page S 10

3.1. Normalised UV/Vis-spectra of compounds 8a, 9a and $\mathbf{7}$ (Figure 2)

Page S 10

3.2. Comparison of the chromophores of compounds $\mathbf{8 a}, 9 \mathbf{a}$, and 7 by Gaussian analysis of their UV/Vis spectra

4. Absolute energies and atom coordinates of structures $\mathbf{8}$ and $\mathbf{9}\left(\mathrm{R}=\mathrm{CH}_{3}\right)$ Page $\mathrm{S} 12$

4.1. Data for structure $8\left(\mathrm{R}=\mathrm{CH}_{3}\right)$

4.2. Data for structure $9\left(\mathrm{R}=\mathrm{CH}_{3}\right)$ 
1. ${ }^{1} \mathrm{H}$ and ${ }^{13} \mathrm{C}$ NMR spectra

1.1. ${ }^{1} \mathrm{H}$ NMR spectrum of compound $\mathbf{8 a}$ (as determined from an enriched mixture of $8 a / 9 a)$

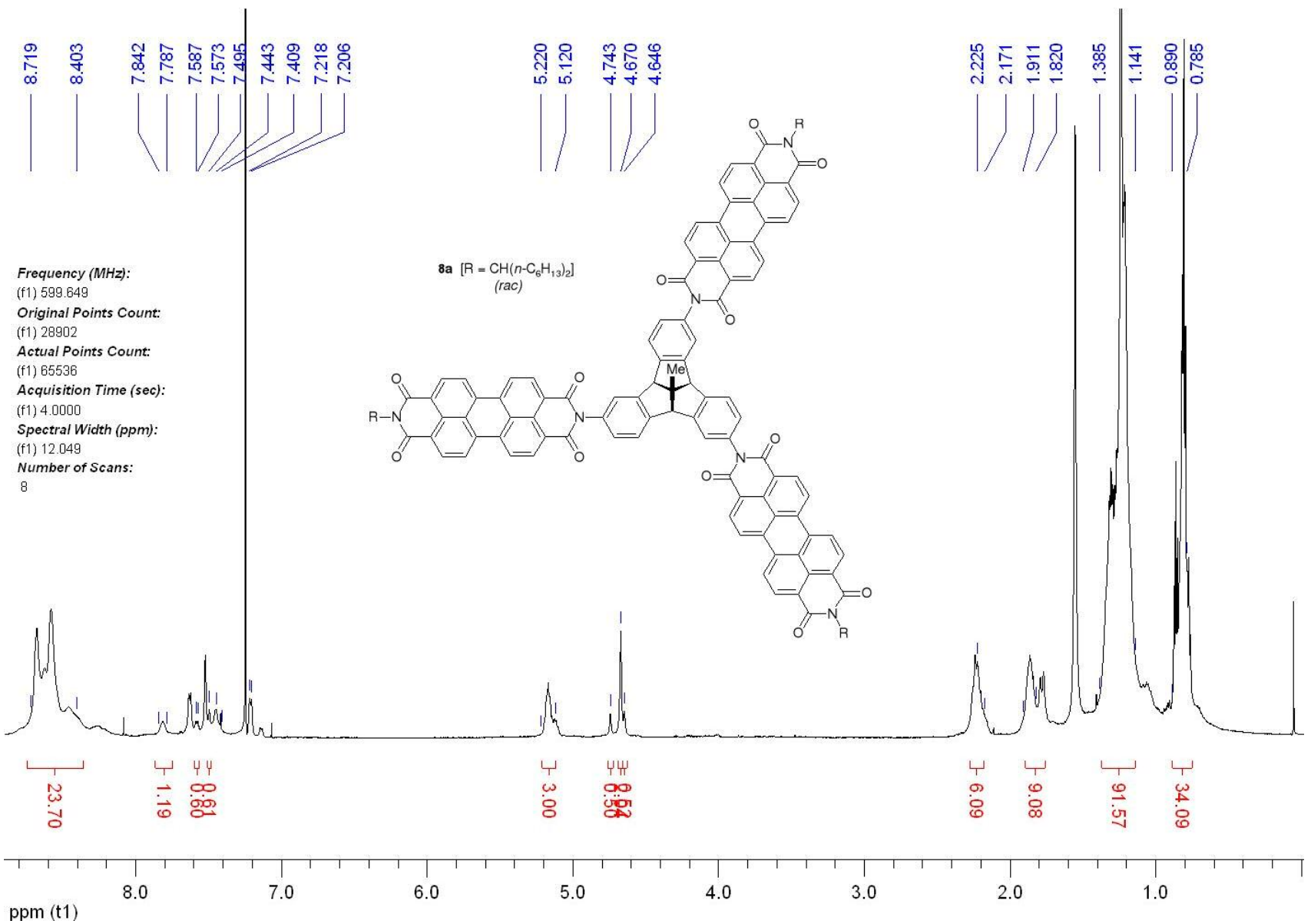


S 3

1.2. ${ }^{13} \mathrm{C}$ NMR spectrum of compound $8 \mathbf{a}\left(151 \mathrm{MHz}, \mathrm{CDCl}_{3}\right.$, as determined from an enriched mixture of $\mathbf{8} \mathbf{a} / \mathbf{9 a} \mathbf{a}$ )

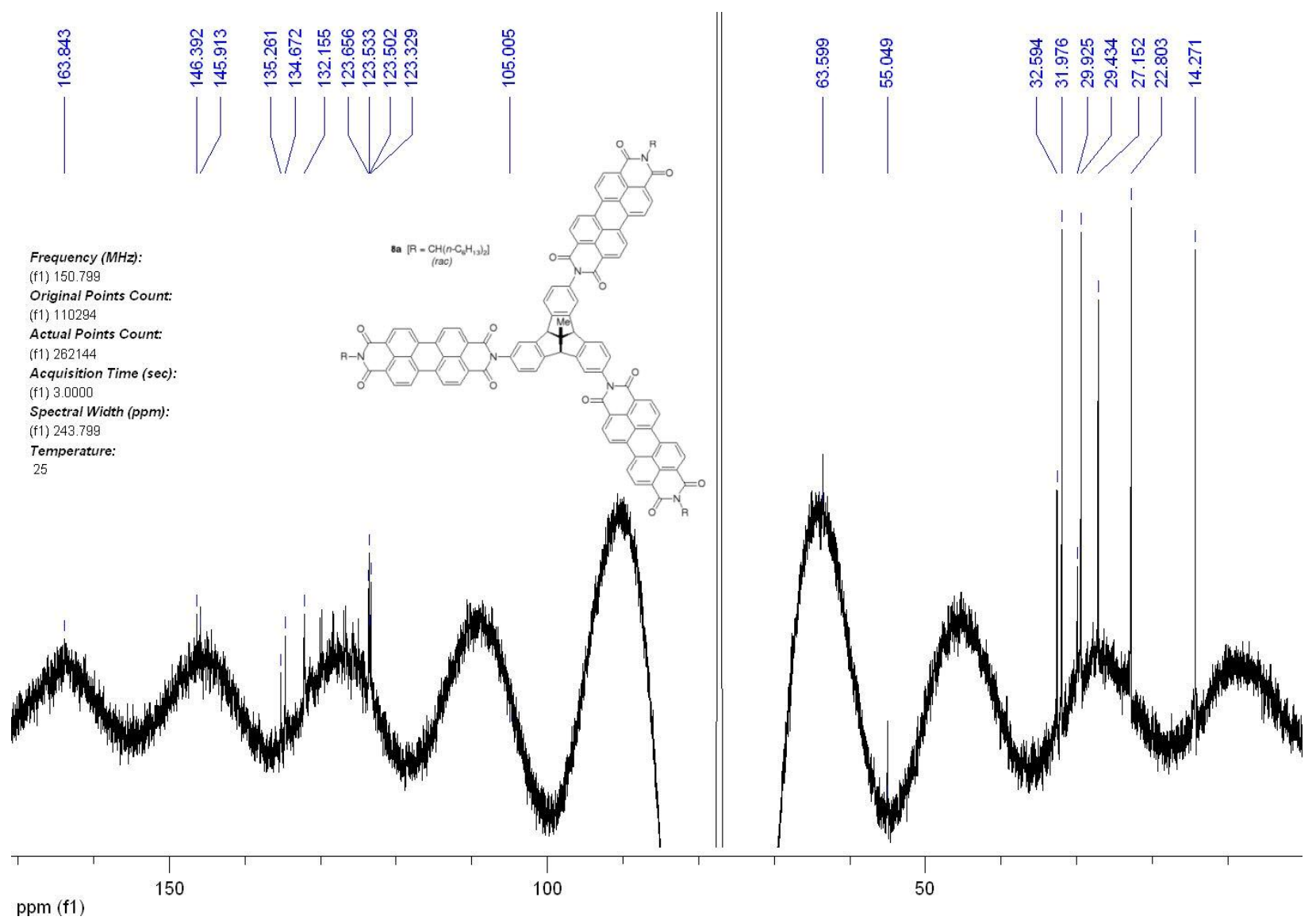


1.3. ${ }^{1} \mathrm{H}$ NMR spectrum of compound $9 \mathrm{a}\left(600 \mathrm{MHz}, \mathrm{CDCl}_{3}\right)$

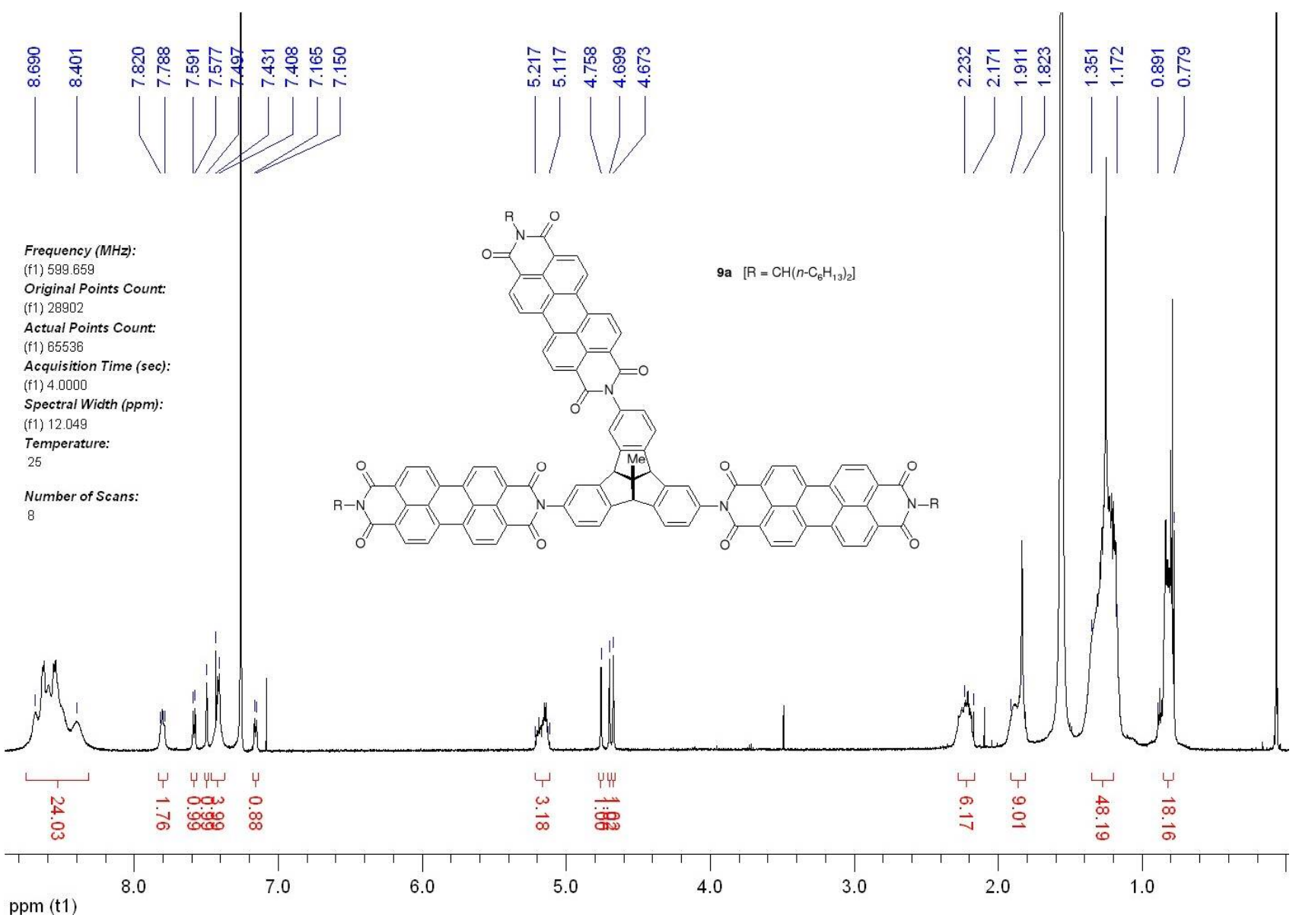


1.4. ${ }^{13} \mathrm{C}$ NMR spectrum of compound $9 \mathbf{a}\left(151 \mathrm{MHz}, \mathrm{CDCl}_{3}\right)$

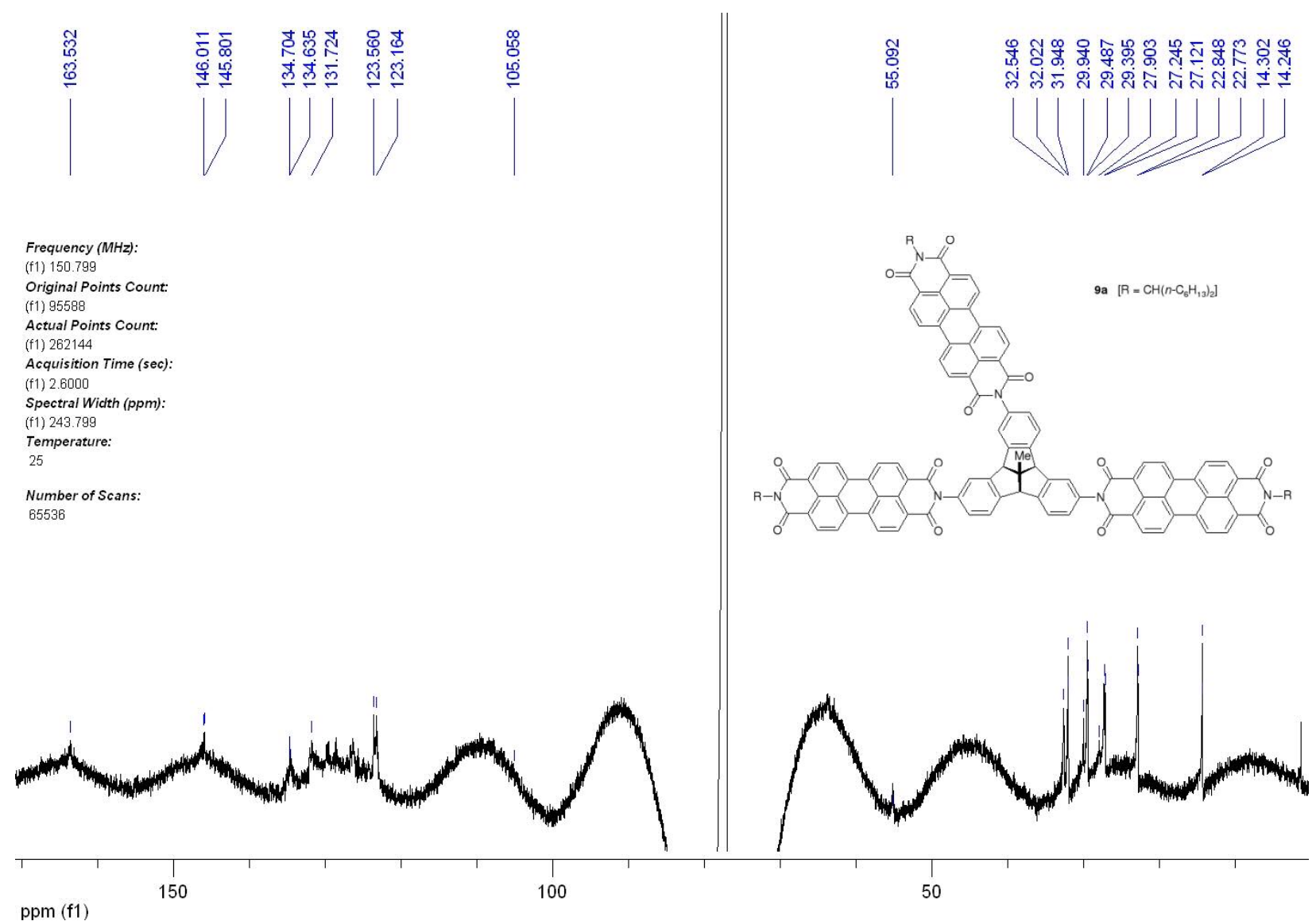


1.5. ${ }^{1} \mathrm{H}$ NMR spectrum of the mixture of $8 \mathbf{b} / 9 \mathbf{b}\left(600 \mathrm{MHz}, \mathrm{CDCl}_{3}\right)$

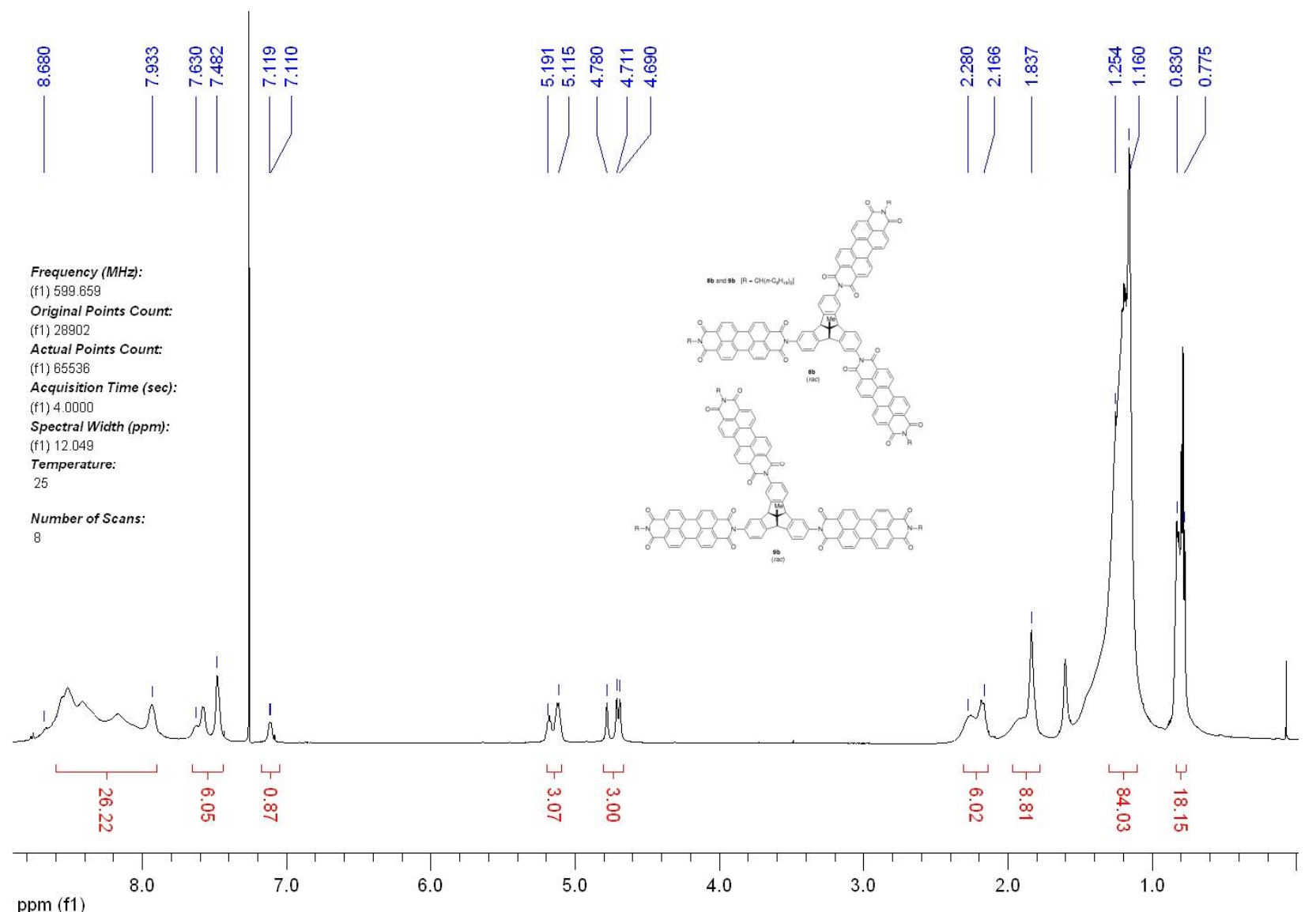


1.6. ${ }^{13} \mathrm{C}$ NMR spectrum of the mixture of $8 \mathbf{b} / 9 \mathbf{b}\left(151 \mathrm{MHz}, \mathrm{CDCl}_{3}\right)$

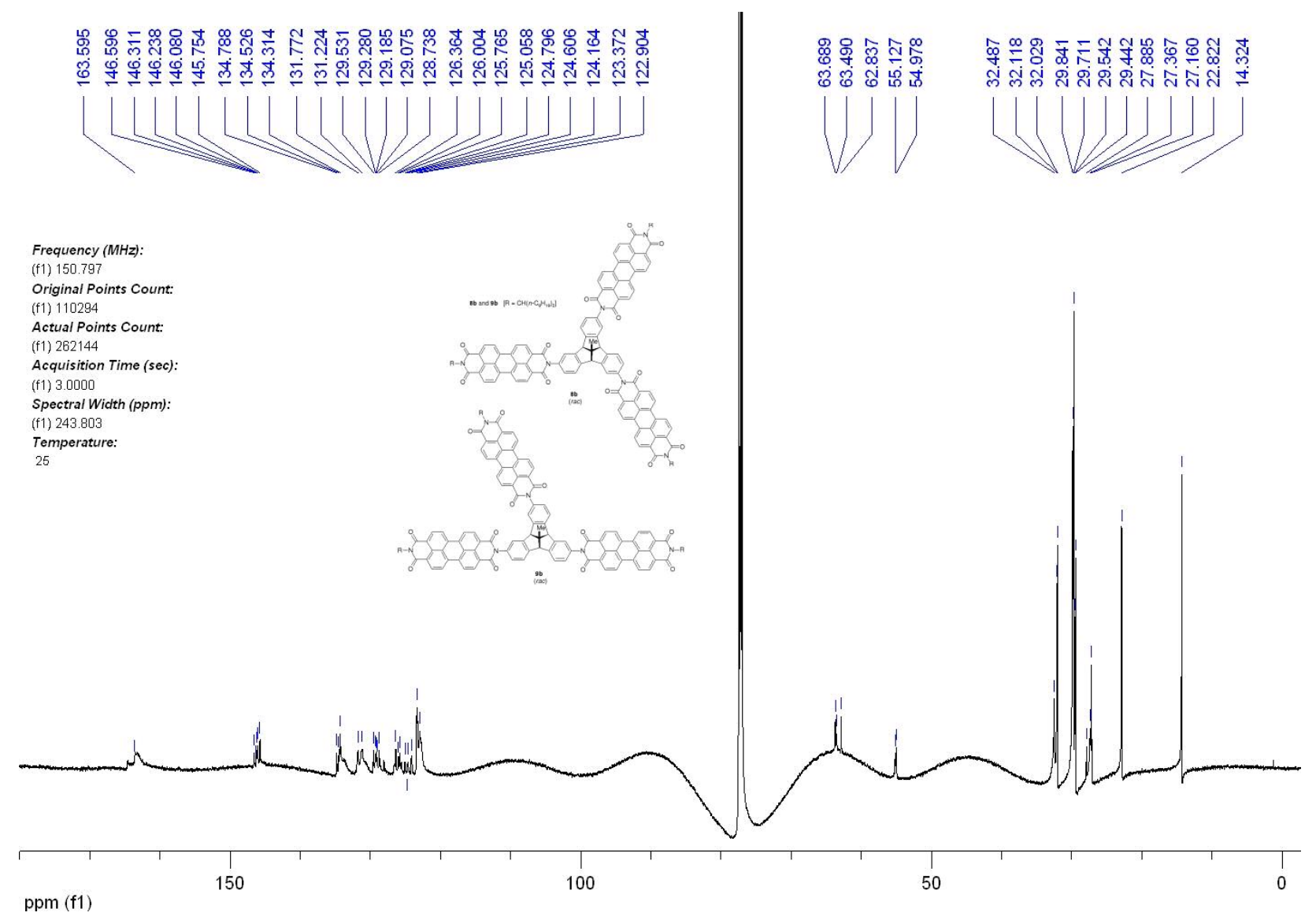


2. Mass spectrometric data of compound $\mathbf{8 a}$

2.1 MALDI-TOF mass spectrum of $8 \mathbf{a}$ at resolution $M / \Delta M=1,500)$, matrix $D C T B^{a}$ with $\mathrm{CHCl}_{3}$ used for preparation. Complete spectrum (top) and molecular ion range (bottom). The peak at $\Delta(\mathrm{m} / \mathrm{z})+16$ is supposed to be due to an artefact.

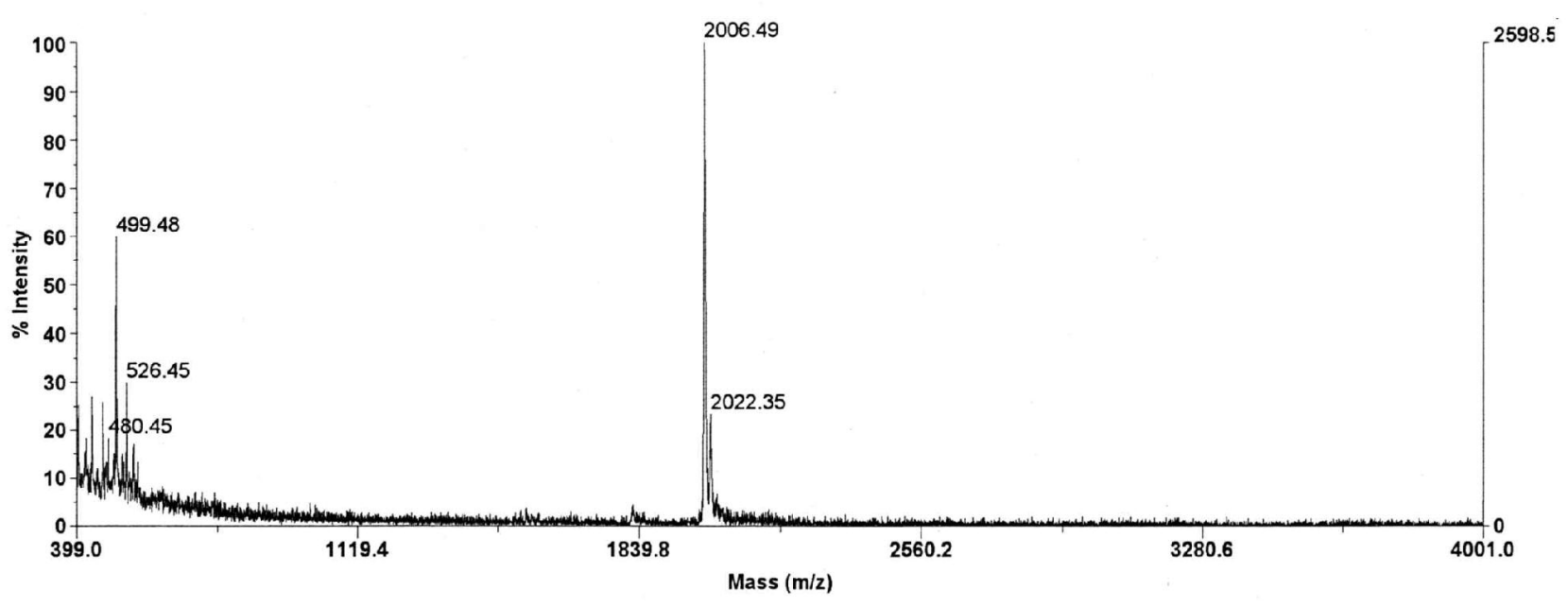

Voyager Spec \#1[BP = 2006.1, 2599]

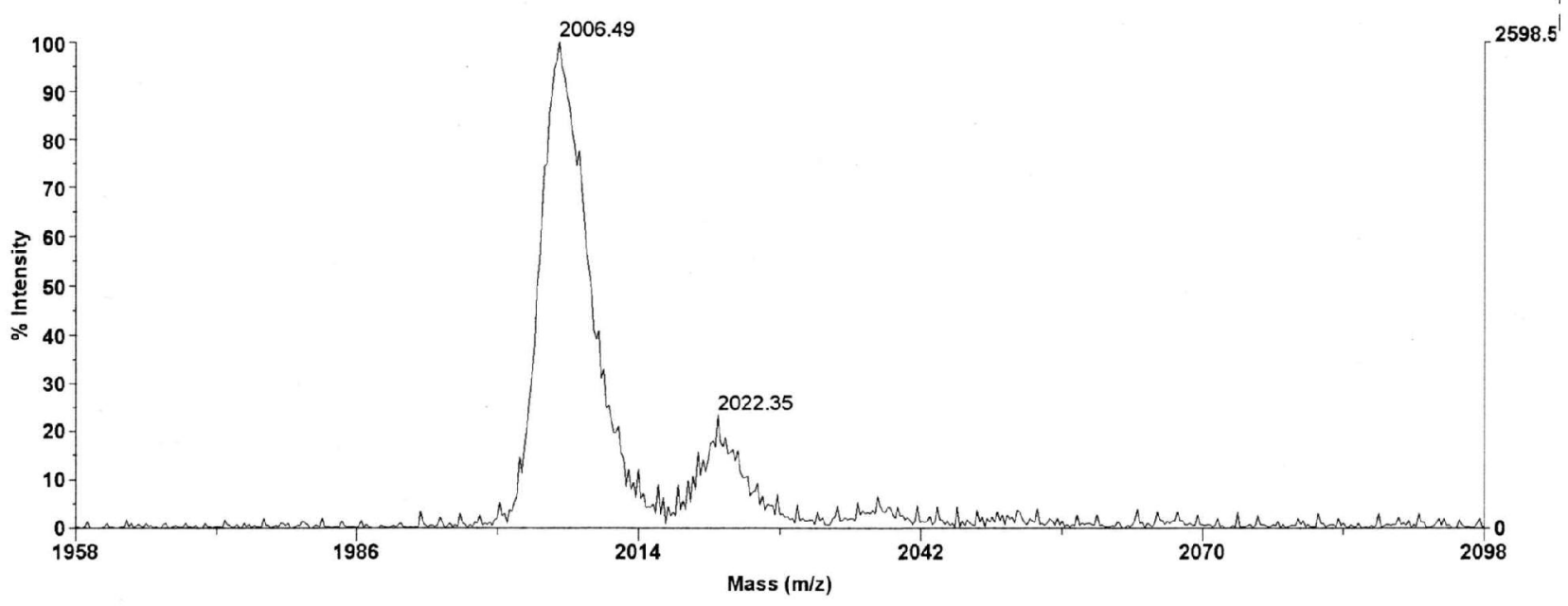

(a) Matrix: "DCTB" is [(2E)-3-(4-tert-butylphenyl)-2-methylprop-2-enylidene]malononitrile. 
2.2 MALDI-FT-ICR accurate mass measurement of $\mathbf{8 a}$ at high resolution $(\mathrm{M} / \Delta \mathrm{M}>$ 20,500), matrix DCTB with $\mathrm{CHCl}_{3}$ used for preparation. Only the molecular peak cluster $\left([\mathrm{M}]^{*+}\right.$ and $\left.[\mathrm{M}-\mathrm{H}]^{+}\right)$is shown. The peak cluster at $\Delta(\mathrm{m} / \mathrm{z})+16$ is supposed to be due to an artefact.

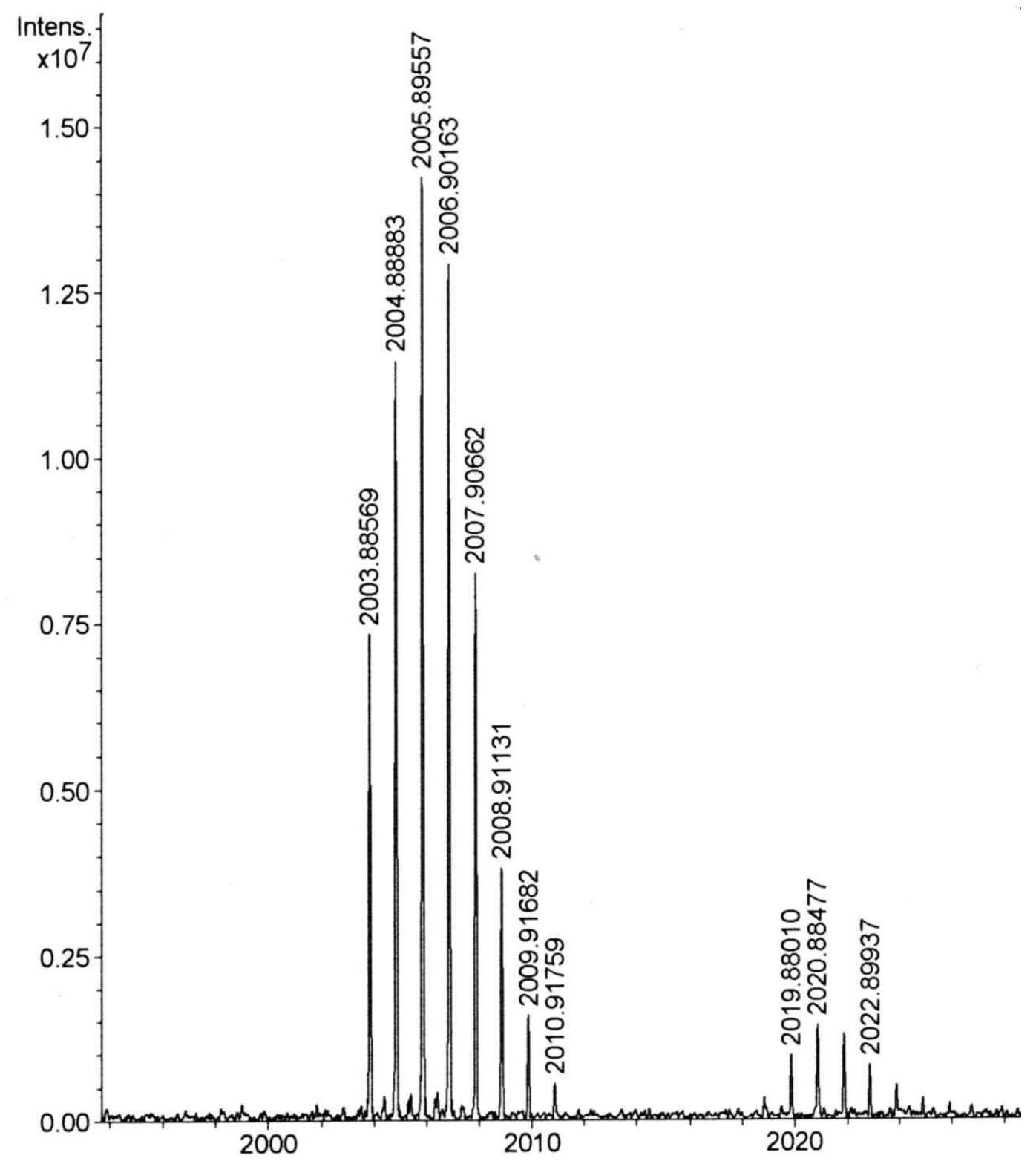


3. Comparison of the chromophores of compounds $\mathbf{8} \mathbf{a}, \mathbf{9 a}$, and $\mathbf{7}$ by Gaussian analysis of their UV/Vis spectra

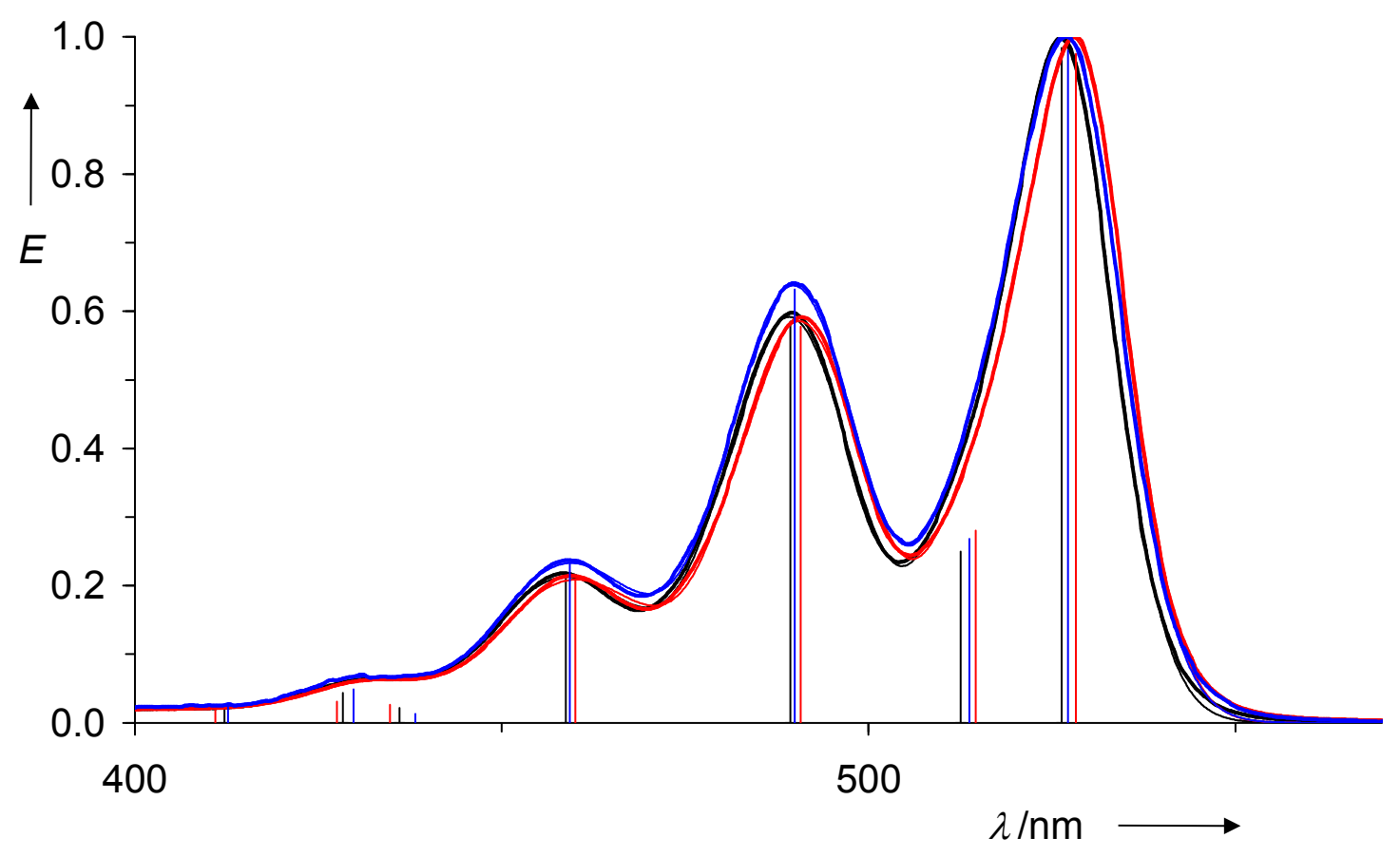

Figure 2. Normalised UV/Vis-spectra of $\mathbf{8 a}$ (red), 9a (blue) and $\mathbf{7}$ (black) in chloroform (thick lines) and calculated spectra from Gaussian analysis (thin lines, nearly covered by the thick lines). Bars: calculated positions and intensities of the Gaussian bands. 
Table I. Gaussian analysis of the UV/Vis spectra of $\mathbf{8 a}, \mathbf{9 a}$, and $\mathbf{7}$ in chloroform.

\begin{tabular}{|c|c|c|c|}
\hline $\begin{array}{l}\text { Gaussian } \\
\text { parameters }^{[a, b]}\end{array}$ & $8 a$ & $9 a$ & 7 \\
\hline$\overline{\lambda_{\max }(1)}$ & 528.3 & 527.2 & 526.3 \\
\hline $2 \sigma^{2}(1)$ & 0.121 & 0.135 & 0.123 \\
\hline$E_{\max }(1)$ & 0.976 & 0.980 & 0.984 \\
\hline$\lambda_{\max }(2)$ & 514.6 & 513.8 & 512.5 \\
\hline $2 \sigma^{2}(2)$ & 0.097 & 0.095 & 0.085 \\
\hline$E_{\max }(2)$ & 0.280 & 0.268 & 0.250 \\
\hline$\lambda_{\max }(3)$ & 490.8 & 489.9 & 489.4 \\
\hline $2 \sigma^{2}(3)$ & 0.272 & 0.286 & 0.268 \\
\hline$E_{\max }(3)$ & 0.578 & 0.632 & 0.586 \\
\hline$\lambda_{\max }(4)$ & 460.0 & 459.3 & 458.7 \\
\hline $2 \sigma^{2}(4)$ & 0.562 & 0.505 & 0.526 \\
\hline$E_{\max }(4)$ & 0.208 & 0.231 & 0.212 \\
\hline$\lambda_{\max }(5)$ & 434.8 & 438.2 & 436.0 \\
\hline $2 \sigma^{2}(5)$ & 0.160 & 0.076 & 0.127 \\
\hline$E_{\max }(5)$ & 0.026 & 0.013 & 0.022 \\
\hline$\lambda_{\max }(6)$ & 427.5 & 429.8 & 428.4 \\
\hline $2 \sigma^{2}(6)$ & 0.313 & 0.253 & 0.252 \\
\hline$E_{\max }(6)$ & 0.031 & 0.049 & 0.044 \\
\hline$\lambda_{\max }(7)$ & 410.9 & 412.7 & 412.2 \\
\hline $2 \sigma^{2}(7)$ & 1.790 & 1.768 & 0.777 \\
\hline$E_{\max }(7)$ & 0.018 & 0.023 & 0.020 \\
\hline
\end{tabular}


4. Atom coordinates and absolute energies of structures $\mathbf{8}\left(\mathrm{R}=\mathrm{CH}_{3}\right)$ and $\mathbf{9}\left(\mathrm{R}=\mathrm{CH}_{3}\right)$

\subsection{Data for structure $8\left(\mathrm{R}=\mathrm{CH}_{3}\right)$}

Summary of AM1 calculation (version 6.00)

$\mathrm{C}_{98} \mathrm{H}_{48} \mathrm{~N}_{6} \mathrm{O}_{12}$

$\mathrm{T}=300000$ AM1 nointer $\mathrm{xyz}$ precise

Ram194a_0.mop

Herberts test was satisfied in BFGS

SCF field was achieved

Heat of formation $=67.189846 \mathrm{kcal} / \mathrm{mol}$

Electronic energy $=-270316.772016 \mathrm{eV}$

Core-core repulsion $=252030.015287 \mathrm{eV}$

Gradient norm $=6.735620$

Dipole $=0.84011$ Debye

No. of filled levels $=271$

Ionization potential $=8.887455 \mathrm{Ev}$

Molecular weight $=1501.490$

SCF calculations $=417$

Computation time $=1 \mathrm{~h} 7 \mathrm{~min} 15.760 \mathrm{~s}$

Parameters: $\mathrm{T}=300000$ AM1 nointer xyz precise

Name: RAM194a_0.Mop

Final geometry:

Table II. Calculated atomic coordinates and charges of $\mathbf{8}$.

$\begin{array}{lllll}\text { Atom } & \mathrm{x} & \mathrm{y} & \mathrm{z} & \text { charge } \\ \mathrm{C} & .0000000 & .000000 & .000000 & -.0834 \\ \mathrm{C} & 1.4158129 & .000000 & .000000 & .0426 \\ \mathrm{C} & 1.4088423 & 119.660408 & .000000 & -.1099 \\ \mathrm{C} & 1.3971052 & 120.445677 & -9.356604 & -.1112 \\ \mathrm{C} & 1.3856492 & 119.285177 & 1.988891 & -.0639 \\ \mathrm{C} & 1.3850416 & 118.547385 & 9.178045 & -.0634 \\ \mathrm{~N} & 1.4327859 & 120.343847 & 176.091382 & -.2752 \\ \mathrm{C} & 1.4962787 & 127.986604 & -171.927830 & -.0199 \\ \mathrm{C} & 1.5003593 & 112.395792 & 69.404364 & -.0619 \\ \mathrm{C} & 1.3858478 & 128.876953 & -64.093886 & -.1053 \\ \mathrm{C} & 1.3988945 & 119.713706 & 175.209972 & -.1132 \\ \mathrm{C} & 1.4084754 & 120.917832 & 2.237863 & .0420 \\ \mathrm{C} & 1.4167081 & 119.093660 & 1.316041 & -.0984 \\ \mathrm{C} & 1.3834791 & 119.076326 & -4.731133 & -.0634 \\ \mathrm{C} & 1.4991224 & 127.493728 & 175.126703 & -.0183 \\ \mathrm{C} & 1.4961371 & 126.862842 & -171.898598 & -.0205 \\ \mathrm{C} & 1.5670815 & 105.135968 & 179.770427 & -.0507 \\ \mathrm{C} & 1.4973803 & 113.007179 & -60.751621 & -.0637 \\ \mathrm{C} & 1.4113662 & 111.419204 & -108.188546 & -.0641 \\ \mathrm{C} & 1.3857019 & 120.047645 & 174.423899 & -.0980 \\ \mathrm{C} & 1.3969910 & 119.433008 & .983708 & -.1093 \\ \mathrm{C} & 1.4090599 & 120.786465 & 2.227634 & .0398 \\ \mathrm{C} & 1.3843766 & 127.401215 & 70.131037 & -.1012 \\ \mathrm{~N} & 1.4332090 & 120.204729 & 177.954932 & -.2747\end{array}$




\begin{tabular}{|c|c|c|c|c|}
\hline$N$ & 1.4340060 & 121.267420 & -176.599316 & -.2733 \\
\hline C & 1.5034682 & 112.208163 & 120.665497 & -.2011 \\
\hline C & 1.4131247 & 118.604716 & -40.705750 & .3590 \\
\hline C & 1.4850920 & 118.616923 & 174.088447 & -.1213 \\
\hline C & 1.4186638 & 119.888394 & 9.248852 & .0154 \\
\hline C & 1.4186467 & 120.082763 & -4.222828 & -.1211 \\
\hline C & 1.4120967 & 119.411003 & 138.933147 & .3609 \\
\hline C & 1.4121836 & 119.843266 & -39.253897 & .3605 \\
\hline C & 1.4868199 & 118.704306 & 176.674851 & -.1221 \\
\hline C & 1.4188161 & 120.478642 & 3.083332 & .0166 \\
\hline C & 1.4175543 & 119.748940 & -1.259347 & -.1227 \\
\hline C & 1.4146182 & 118.448595 & 138.514115 & .3597 \\
\hline C & 1.4100307 & 119.161866 & -47.120800 & .3617 \\
\hline C & 1.4862133 & 118.493063 & 179.795008 & -.1170 \\
\hline C & 1.4193454 & 120.414172 & 2.001350 & .0154 \\
\hline C & 1.4192734 & 119.773900 & -1.610646 & -.1180 \\
\hline C & 1.4114507 & 118.452393 & 133.721869 & .3608 \\
\hline C & 1.4180904 & 120.140766 & 178.086641 & -.0318 \\
\hline C & 1.4310319 & 119.067481 & -177.946024 & .0050 \\
\hline C & 1.3865163 & 119.208833 & -2.536453 & -.1259 \\
\hline C & 1.3795206 & 120.003375 & 179.838774 & -.0445 \\
\hline C & 1.3794545 & 119.639792 & -179.006359 & -.0474 \\
\hline C & 1.4092266 & 120.163615 & -179.015108 & -.1285 \\
\hline C & 1.3862987 & 121.493623 & .158994 & .0052 \\
\hline C & 1.4156869 & 119.956055 & 174.899931 & -.0327 \\
\hline C & 1.4303622 & 118.993162 & -173.112563 & .0078 \\
\hline C & 1.3860373 & 119.360596 & -5.944097 & -.1277 \\
\hline C & 1.3798715 & 120.086265 & 176.747847 & -.0433 \\
\hline C & 1.3803002 & 120.008250 & -172.608703 & -.0422 \\
\hline C & 1.4101212 & 120.087306 & -179.040768 & -.1267 \\
\hline C & 1.3853184 & 121.235898 & 1.925944 & .0071 \\
\hline C & 1.4588632 & 121.786391 & -179.767151 & .0105 \\
\hline C & 1.4312364 & 118.983720 & -175.596841 & -.0337 \\
\hline C & 1.4309679 & 121.892200 & -2.413117 & .0108 \\
\hline C & 1.4581052 & 121.957681 & -179.835411 & .0081 \\
\hline C & 1.4310663 & 118.446441 & -166.698104 & -.0335 \\
\hline C & 1.4304787 & 121.993044 & -8.118293 & .0082 \\
\hline C & 1.3803927 & 119.727966 & -177.224295 & -.0419 \\
\hline C & 1.4100505 & 120.263109 & 178.467005 & -.1276 \\
\hline C & 1.3862763 & 121.359648 & .964036 & .0089 \\
\hline C & 1.4170070 & 120.206438 & 179.040293 & -.0329 \\
\hline C & 1.4308154 & 118.964251 & -176.390188 & .0084 \\
\hline C & 1.3861030 & 119.384700 & -3.688062 & -.1280 \\
\hline C & 1.3810278 & 120.147708 & 178.522406 & -.0413 \\
\hline C & 1.4589273 & 122.040639 & -176.644290 & .0089 \\
\hline C & 1.4311911 & 118.816133 & -172.943201 & -.0331 \\
\hline C & 1.4307311 & 121.893600 & -1.942207 & .0084 \\
\hline C & 1.4178409 & 119.039700 & 177.503532 & .0175 \\
\hline C & 1.4203765 & 119.981026 & -178.604778 & -.1230 \\
\hline C & 1.3792924 & 120.137843 & -.095808 & -.0414 \\
\hline C & 1.3864258 & 119.284846 & 178.106796 & -.1295 \\
\hline C & 1.3866149 & 121.762734 & 3.984452 & -.1314 \\
\hline C & 1.4091128 & 121.438294 & -178.065884 & -.0421 \\
\hline C & 1.3795106 & 120.139070 & -.158847 & -.1223 \\
\hline C & 1.4155157 & 118.935127 & 171.798208 & .0160 \\
\hline C & 1.4203486 & 119.776188 & -174.004261 & -.1214 \\
\hline C & 1.3796435 & 120.220676 & -3.795820 & -.0419 \\
\hline C & 1.3856310 & 119.426165 & 175.737012 & -.1278 \\
\hline C & 1.3857420 & 122.174452 & 12.245193 & -.1279 \\
\hline C & 1.4103318 & 121.195434 & -175.514351 & -.0414 \\
\hline C & 1.3798511 & 120.065771 & 1.612247 & -.1214 \\
\hline C & 1.4168832 & 119.103139 & 177.812780 & .0165 \\
\hline C & 1.4203964 & 119.858110 & -176.006465 & -.1219 \\
\hline
\end{tabular}




\begin{tabular}{|c|c|c|c|c|}
\hline C & 1.3795652 & 120.166519 & .640654 & -.0419 \\
\hline C & 1.3854977 & 119.405753 & 173.863945 & -.1283 \\
\hline C & 1.3860778 & 121.944317 & 7.417814 & -.1276 \\
\hline C & 1.4099364 & 121.398077 & 179.868454 & -.0416 \\
\hline C & 1.3797830 & 120.137971 & 1.050263 & -.1211 \\
\hline C & 1.4841950 & 119.799169 & 179.589851 & .3566 \\
\hline $\mathrm{N}$ & 1.4044106 & 118.478619 & -179.147432 & -.3191 \\
\hline C & 1.4051706 & 122.678478 & -1.285986 & .3541 \\
\hline C & 1.4836520 & 120.112944 & 177.535318 & .3561 \\
\hline $\mathrm{N}$ & 1.4057438 & 118.377839 & -173.014992 & -.3206 \\
\hline C & 1.4061727 & 122.692303 & -6.731640 & .3553 \\
\hline C & 1.4834324 & 119.998410 & -179.183854 & .3553 \\
\hline $\mathrm{N}$ & 1.4056396 & 118.513373 & -178.015868 & -.3193 \\
\hline C & 1.4049306 & 122.678101 & .941102 & .3555 \\
\hline 0 & 1.2444274 & 119.924657 & 179.791485 & -.3292 \\
\hline 0 & 1.2442745 & 121.658977 & 6.240269 & -.3291 \\
\hline 0 & 1.2452545 & 121.521495 & .852999 & -.3321 \\
\hline 0 & 1.2449991 & 119.604164 & -179.480629 & -.3294 \\
\hline $\mathrm{O}$ & 1.2430438 & 120.845940 & -.971720 & -.3192 \\
\hline 0 & 1.2430738 & 120.847897 & -.160390 & -.3129 \\
\hline 0 & 1.2431770 & 121.148997 & -.183275 & -.3183 \\
\hline 0 & 1.2438286 & 120.941067 & -3.282553 & -.3197 \\
\hline 0 & 1.2451105 & 119.958725 & 176.867185 & -.3311 \\
\hline 0 & 1.2451069 & 121.731708 & 2.379705 & -.3297 \\
\hline $\mathrm{O}$ & 1.2429849 & 121.212199 & -2.256056 & -.3181 \\
\hline $\mathrm{O}$ & 1.2433477 & 121.012518 & -5.955286 & -.3163 \\
\hline C & 1.4448336 & 118.865432 & 174.103313 & -.0851 \\
\hline C & 1.4453490 & 118.503100 & 179.402938 & -.0856 \\
\hline C & 1.4454490 & 119.319010 & -179.816423 & -.0857 \\
\hline $\mathrm{H}$ & 1.1018045 & 120.744731 & -173.833154 & .1596 \\
\hline $\mathrm{H}$ & 1.1010855 & 120.258223 & 175.346421 & .1440 \\
\hline $\mathrm{H}$ & 1.0996641 & 120.143363 & -176.539781 & .1361 \\
\hline $\mathrm{H}$ & 1.1197464 & 111.664773 & -56.273068 & .1173 \\
\hline $\mathrm{H}$ & 1.1001768 & 120.791788 & -2.911362 & .1382 \\
\hline $\mathrm{H}$ & 1.1014040 & 118.632092 & -178.221382 & .1521 \\
\hline $\mathrm{H}$ & 1.1006501 & 121.072383 & 178.384182 & .1506 \\
\hline $\mathrm{H}$ & 1.1202064 & 110.819229 & 65.071196 & .1189 \\
\hline $\mathrm{H}$ & 1.1207864 & 111.310487 & -59.447131 & .1173 \\
\hline $\mathrm{H}$ & 1.1011174 & 120.839916 & -176.816450 & .1469 \\
\hline $\mathrm{H}$ & 1.1018297 & 118.769515 & -178.775208 & .1476 \\
\hline $\mathrm{H}$ & 1.1012835 & 120.118301 & 3.114488 & .1521 \\
\hline $\mathrm{H}$ & 1.1175346 & 110.312343 & 60.710056 & .0817 \\
\hline $\mathrm{H}$ & 1.1170146 & 110.434368 & -59.252781 & .0805 \\
\hline $\mathrm{H}$ & 1.1170504 & 110.442766 & -179.343462 & .0816 \\
\hline $\mathrm{H}$ & 1.1011678 & 121.298720 & -178.840129 & .1464 \\
\hline $\mathrm{H}$ & 1.1038577 & 119.974472 & 179.148486 & .1707 \\
\hline $\mathrm{H}$ & 1.1040039 & 119.936059 & .687460 & .1695 \\
\hline $\mathrm{H}$ & 1.1001150 & 117.205158 & 179.603347 & .1451 \\
\hline $\mathrm{H}$ & 1.1005577 & 121.133642 & -177.542597 & .1461 \\
\hline $\mathrm{H}$ & 1.1040831 & 119.945939 & 179.126272 & 1682 \\
\hline $\mathrm{H}$ & 1.1034390 & 120.002060 & .636438 & 1692 \\
\hline $\mathrm{H}$ & 1.1008369 & 117.873515 & -179.151850 & .1472 \\
\hline $\mathrm{H}$ & 1.1043176 & 119.945873 & -.979388 & .1699 \\
\hline $\mathrm{H}$ & 1.1006708 & 117.443568 & -179.231525 & .1470 \\
\hline $\mathrm{H}$ & 1.1016013 & 121.071753 & -178.267867 & .1470 \\
\hline $\mathrm{H}$ & 1.1041944 & 120.144111 & 179.599123 & .1700 \\
\hline $\mathrm{H}$ & 1.1047974 & 119.817914 & 179.327844 & .1698 \\
\hline $\mathrm{H}$ & 1.1012031 & 121.204707 & -178.509447 & .1468 \\
\hline $\mathrm{H}$ & 1.1003110 & 121.223233 & 1.722990 & .1456 \\
\hline $\mathrm{H}$ & 1.1035971 & 119.847900 & 179.592202 & 1691 \\
\hline $\mathrm{H}$ & 1.1035855 & 119.913074 & 179.796539 & 1692 \\
\hline $\mathrm{H}$ & 1.1013250 & 120.963080 & -178.980593 & .1462 \\
\hline $\mathrm{H}$ & 1.1007717 & 120.947934 & 4.349873 & 1472 \\
\hline
\end{tabular}




$\begin{array}{lllll}\mathrm{H} & 1.1041293 & 119.906346 & -179.224246 & .1692 \\ \mathrm{H} & 1.1041756 & 119.907303 & 177.833625 & .1695 \\ \mathrm{H} & 1.1011313 & 121.125074 & -176.460120 & .1471 \\ \mathrm{H} & 1.1005186 & 121.106709 & .265156 & .1468 \\ \mathrm{H} & 1.1035294 & 119.916629 & -179.068473 & .1693 \\ \mathrm{H} & 1.1214199 & 109.917095 & 94.179508 & .0856 \\ \mathrm{H} & 1.1228670 & 109.680786 & -25.604570 & .1104 \\ \mathrm{H} & 1.1224131 & 109.318117 & -146.143817 & .1074 \\ \mathrm{H} & 1.1225342 & 109.642944 & 154.228527 & .1109 \\ \mathrm{H} & 1.1223437 & 109.480626 & 33.802021 & .1081 \\ \mathrm{H} & 1.1223978 & 109.841459 & -85.915612 & .0851 \\ \mathrm{H} & 1.1225205 & 109.361219 & -139.256569 & .1030 \\ \mathrm{H} & 1.1220368 & 109.665348 & 101.517939 & .0857 \\ \mathrm{H} & 1.1211102 & 109.955472 & -18.707101 & .1157\end{array}$

\subsection{Data for structure $9\left(\mathrm{R}=\mathrm{CH}_{3}\right)$}

Summary of $\quad$ AM1 calculation (version 6.00)

$\mathrm{C}_{98} \mathrm{H}_{48} \mathrm{~N}_{6} \mathrm{O}_{12}$

$\mathrm{T}=300000$ AM1 nointer $x y z$ precise

Ram194b_0.mop

Gradient test not passed, but further work not justified

SCF field was achieved

Heat of formation $=67.847349 \mathrm{kcal} / \mathrm{mol}$

Electronic energy $=-264686.007798 \mathrm{eV}$

Core-core repulsion $=246399.279580 \mathrm{eV}$

Gradient norm $=10.610688$

Dipole $=0.19640$ Debye

No. of filled levels $=271$

Ionization potential $=8.862465 \mathrm{eV}$

Molecular weight $=1501.490$

SCF calculations $=315$

Computation time $=3 \mathrm{~h} 48 \mathrm{~min} 8.540 \mathrm{~s}$

Parameters: $\mathrm{T}=300000$ AM1 nointer xyz precise

Name: RAM194b_0.mop

Final geometry:

Table III. Calculated atomic coordinates and charges of $\mathbf{9}$.

$\begin{array}{ll}\text { Atom } & \mathrm{x} \\ \mathrm{C} & .0000000 \\ \mathrm{C} & 1.3973407 \\ \mathrm{C} & 1.4096880 \\ \mathrm{C} & 1.4165980 \\ \mathrm{C} & 1.3827765 \\ \mathrm{C} & 1.3860007 \\ \mathrm{C} & 1.4971339\end{array}$

$\mathrm{y}$

000000

.000000

120.030076

119.581069

119.005789

120.164064

126.928056 z

.000000

.000000

.000000

$-4.621753$

$-2.423957$

7.624619

$-167.830814$ charge

-.1017

$-.1080$

.0384

$-.1000$

$-.0662$

$-.0616$

$-.0180$ 


\begin{tabular}{|c|c|c|c|c|}
\hline C & 1.5005978 & 111.452451 & 67.701982 & -.0658 \\
\hline C & 1.3852705 & 128.546444 & -62.776862 & -.0966 \\
\hline C & 1.3972224 & 119.591384 & 174.915629 & -.1117 \\
\hline C & 1.4089763 & 120.971620 & 1.683158 & .0406 \\
\hline C & 1.4141368 & 119.219365 & .263969 & -.1000 \\
\hline C & 1.3849223 & 119.025358 & -2.635106 & -.0641 \\
\hline C & 1.4990076 & 128.995393 & 173.309080 & -.0185 \\
\hline C & 1.4964071 & 126.978131 & -173.084405 & -.0197 \\
\hline C & 1.5664129 & 104.847133 & -179.227979 & -.0509 \\
\hline C & 1.4967446 & 114.018261 & -59.944194 & -.0630 \\
\hline C & 1.4129635 & 111.667609 & -108.633691 & -.0628 \\
\hline C & 1.3853982 & 120.094521 & 176.115147 & -.0988 \\
\hline C & 1.3964875 & 119.464123 & 231288 & -.1108 \\
\hline C & 1.4080154 & 120.731384 & 2.172288 & .0391 \\
\hline C & 1.3831417 & 127.272009 & 70.804258 & -.1002 \\
\hline C & 1.5038321 & 112.350912 & 119.019597 & -.2009 \\
\hline C & 2.4498874 & 95.830507 & 161.004334 & .3605 \\
\hline C & 1.4843680 & 148.676836 & 134.484440 & -.1223 \\
\hline C & 1.4183842 & 120.099410 & 16.149394 & .0160 \\
\hline C & 1.4182944 & 120.019811 & -4.403919 & -.1224 \\
\hline C & 1.4839493 & 120.005553 & -4.670279 & .3603 \\
\hline C & 1.4152666 & 120.076533 & 175.022382 & -.0325 \\
\hline C & 1.4296954 & 119.012861 & -174.096824 & .0076 \\
\hline C & 1.3864849 & 119.389805 & -4.674713 & -.1269 \\
\hline C & 1.3796373 & 120.251629 & 176.683981 & -.0421 \\
\hline C & 1.3796110 & 119.796652 & -165.725340 & -.0427 \\
\hline C & 1.4093068 & 120.063491 & -178.990643 & -.1272 \\
\hline C & 1.3855149 & 121.225170 & 2.124152 & .0078 \\
\hline C & 1.4573979 & 121.934893 & -179.222525 & .0088 \\
\hline C & 1.4312865 & 118.840348 & -170.676700 & -.0333 \\
\hline C & 1.4302729 & 121.922422 & -4.595813 & .0088 \\
\hline C & 1.4167063 & 119.025670 & 175.518798 & .0168 \\
\hline C & 1.4201304 & 119.803447 & -175.967102 & -.1222 \\
\hline C & 1.3800806 & 120.237282 & -.701779 & -.0415 \\
\hline C & 1.3858812 & 119.444516 & 175.010434 & -.1282 \\
\hline C & 1.3854132 & 121.786860 & 9.006879 & -.1285 \\
\hline C & 1.4090758 & 121.284940 & -177.782989 & -.0417 \\
\hline C & 1.3797921 & 120.125440 & .657707 & -.1218 \\
\hline C & 1.4827246 & 119.957074 & 179.897656 & .3555 \\
\hline $\mathrm{N}$ & 1.4058730 & 118.495569 & -178.140868 & -.3199 \\
\hline C & 1.4047411 & 122.806850 & -.066800 & .3559 \\
\hline $\mathrm{O}$ & 1.2444196 & 121.696465 & 2.130811 & -.3296 \\
\hline 0 & 1.2446843 & 119.898416 & 177.808732 & -.3311 \\
\hline 0 & 1.2428326 & 90.500247 & -39.539906 & -.3162 \\
\hline 0 & 1.2438099 & 120.262338 & -171.184312 & -.3184 \\
\hline C & 1.4452172 & 118.442690 & 179.083727 & -.0864 \\
\hline $\mathrm{N}$ & 1.4120857 & 118.449195 & 9.054253 & -.2746 \\
\hline C & 2.4544918 & 97.481878 & 161.125043 & .3602 \\
\hline C & 1.4845558 & 149.010973 & 138.958372 & -.1219 \\
\hline C & 1.4188754 & 120.305487 & 8.022352 & .0161 \\
\hline C & 1.4181172 & 119.953003 & -3.390700 & -.1219 \\
\hline C & 1.4849358 & 120.015640 & -3.658272 & .3597 \\
\hline C & 1.4160270 & 120.131862 & 177.093127 & -.0324 \\
\hline C & 1.4302375 & 119.136206 & -175.639634 & .0076 \\
\hline C & 1.3856697 & 119.298435 & -3.854610 & -.1270 \\
\hline C & 1.3803154 & 120.101564 & 177.848904 & -.0426 \\
\hline C & 1.3792335 & 119.600365 & -172.611108 & -.0425 \\
\hline C & 1.4092604 & 120.022701 & 179.607966 & -.1273 \\
\hline C & 1.3860828 & 121.283757 & 1.368124 & .0074 \\
\hline C & 1.4574427 & 121.959011 & -177.438300 & .0088 \\
\hline C & 1.4308295 & 118.968593 & -172.101778 & -.0332 \\
\hline C & 1.4302131 & 121.969543 & -3.725633 & .0091 \\
\hline C & 1.4170333 & 118.959516 & 176.626101 & .0168 \\
\hline
\end{tabular}




\begin{tabular}{|c|c|c|c|c|}
\hline C & 1.4206964 & 119.947121 & -177.049376 & -.1219 \\
\hline C & 1.3794194 & 120.037589 & .106218 & -.0420 \\
\hline C & 1.3864352 & 119.363770 & 175.450927 & -.1280 \\
\hline C & 1.3859845 & 121.581428 & 8.442515 & -.1288 \\
\hline C & 1.4086301 & 121.262645 & -178.620241 & -.0418 \\
\hline C & 1.3802412 & 120.097381 & .884079 & -.1219 \\
\hline C & 1.4829941 & 119.809943 & 178.750799 & .3544 \\
\hline $\mathrm{N}$ & 1.4055746 & 118.600324 & 179.563738 & -.3197 \\
\hline C & 1.4044555 & 122.671034 & 1.631975 & .3568 \\
\hline 0 & 1.2441313 & 121.635230 & -.215782 & -.3293 \\
\hline $\mathrm{O}$ & 1.2441800 & 119.926909 & 177.784451 & -.3313 \\
\hline 0 & 1.2430881 & 90.245053 & -38.105555 & -.3189 \\
\hline 0 & 1.2425123 & 120.218088 & -170.977768 & -.3159 \\
\hline C & 1.4453112 & 117.979960 & 179.709931 & -.0854 \\
\hline $\mathrm{N}$ & 1.4105435 & 30.562902 & 144.461869 & -.2752 \\
\hline $\mathrm{N}$ & 1.4340288 & 120.508997 & -178.321482 & -.2738 \\
\hline C & 1.4124794 & 118.923790 & -38.519390 & .3596 \\
\hline C & 1.4852554 & 118.654623 & 172.596273 & -.1219 \\
\hline C & 1.4180303 & 120.219597 & 7.349992 & .0154 \\
\hline C & 1.4186804 & 119.925346 & -3.269258 & -.1207 \\
\hline C & 1.4139236 & 119.173794 & 138.519527 & .3605 \\
\hline C & 1.4163039 & 120.029123 & 176.669519 & -.0329 \\
\hline C & 1.4304192 & 119.111768 & -174.035793 & .0072 \\
\hline C & 1.3854130 & 119.162355 & -5.804405 & -.1270 \\
\hline C & 1.3803832 & 119.849259 & 177.717346 & -.0424 \\
\hline C & 1.3802844 & 119.785005 & -173.434571 & -.0431 \\
\hline C & 1.4101637 & 120.118685 & 179.283021 & -.1269 \\
\hline C & 1.3852763 & 121.289661 & 1.948103 & .0070 \\
\hline C & 1.4582815 & 122.070119 & -178.496935 & .0086 \\
\hline C & 1.4307428 & 118.655063 & -167.573373 & -.0338 \\
\hline C & 1.4306448 & 121.784131 & -7.572591 & .0088 \\
\hline C & 1.4162961 & 119.041961 & 172.646873 & .0163 \\
\hline C & 1.4209622 & 119.904606 & -174.993093 & -.1211 \\
\hline C & 1.3796970 & 119.963261 & -2.776665 & -.0418 \\
\hline C & 1.3856761 & 119.259753 & 175.833004 & -.1280 \\
\hline C & 1.3857082 & 122.020429 & 12.172372 & -.1285 \\
\hline C & 1.4101519 & 121.177424 & -175.920486 & -.0412 \\
\hline C & 1.3802064 & 120.134800 & 1.459766 & -.1222 \\
\hline C & 1.4838604 & 119.984998 & 176.675087 & .3569 \\
\hline $\mathrm{N}$ & 1.4054679 & 118.498351 & -176.489120 & -.3202 \\
\hline C & 1.4064348 & 122.723264 & -3.466938 & .3553 \\
\hline $\mathrm{O}$ & 1.2446242 & 121.507128 & 3.039468 & -.3314 \\
\hline 0 & 1.2443086 & 119.718473 & 179.648064 & -.3288 \\
\hline $\mathrm{O}$ & 1.2426664 & 120.990025 & -7.178680 & -.3177 \\
\hline 0 & 1.2428668 & 121.183215 & .853819 & -.3169 \\
\hline C & 1.4454636 & 119.011121 & 175.214976 & -.0866 \\
\hline $\mathrm{H}$ & 1.1019393 & 119.193402 & -177.258241 & .1457 \\
\hline $\mathrm{H}$ & 1.1016487 & 119.076447 & 176.991152 & .1522 \\
\hline $\mathrm{H}$ & 1.1005893 & 120.946235 & -178.158071 & .1469 \\
\hline $\mathrm{H}$ & 1.1190911 & 111.863440 & -57.852593 & .1170 \\
\hline $\mathrm{H}$ & 1.1013660 & 120.821580 & -2.365302 & .1468 \\
\hline $\mathrm{H}$ & 1.1017456 & 118.440023 & -178.519518 & .1495 \\
\hline $\mathrm{H}$ & 1.1006479 & 120.977630 & -179.869240 & .1505 \\
\hline $\mathrm{H}$ & 1.1211171 & 110.462120 & 65.901524 & 1160 \\
\hline $\mathrm{H}$ & 1.1199981 & 111.215508 & -61.635295 & .1168 \\
\hline $\mathrm{H}$ & 1.0997377 & 121.017658 & -177.617408 & .1449 \\
\hline $\mathrm{H}$ & 1.1021911 & 118.697483 & -178.783713 & .1488 \\
\hline $\mathrm{H}$ & 1.1013101 & 120.063292 & 2.166778 & .1502 \\
\hline $\mathrm{H}$ & 1.1171282 & 110.379233 & -178.435971 & .0815 \\
\hline $\mathrm{H}$ & 1.1161068 & 110.502628 & 61.480568 & .0806 \\
\hline $\mathrm{H}$ & 1.1166659 & 110.260636 & -58.519862 & .0806 \\
\hline $\mathrm{H}$ & 1.1004107 & 121.106080 & -177.883373 & .1470 \\
\hline $\mathrm{H}$ & 1.1046034 & 120.042904 & 179.789376 & .1699 \\
\hline
\end{tabular}




$\begin{array}{lllll}\mathrm{H} & 1.1042478 & 120.060812 & 1.085006 & .1692 \\ \mathrm{H} & 1.1005614 & 117.603607 & -178.655781 & .1470 \\ \mathrm{H} & 1.1032368 & 119.930158 & 178.895039 & .1694 \\ \mathrm{H} & 1.1002706 & 121.110385 & -177.438860 & .1472 \\ \mathrm{H} & 1.1015650 & 121.130412 & 2.606945 & .1469 \\ \mathrm{H} & 1.1043557 & 120.012737 & -179.584941 & .1693 \\ \mathrm{H} & 1.1230796 & 109.570682 & 155.871888 & .1124 \\ \mathrm{H} & 1.1230383 & 109.527582 & 35.328622 & .1070 \\ \mathrm{H} & 1.1226817 & 109.784684 & -84.324501 & .0851 \\ \mathrm{H} & 1.1009870 & 120.974275 & -178.606634 & .1469 \\ \mathrm{H} & 1.1031039 & 120.046701 & 179.170179 & .1688 \\ \mathrm{H} & 1.1032236 & 119.938415 & .000000 & .1698 \\ \mathrm{H} & 1.1016428 & 117.537302 & -178.578618 & .1470 \\ \mathrm{H} & 1.1036365 & 119.814036 & 178.853196 & .1691 \\ \mathrm{H} & 1.1005670 & 121.165528 & -176.978566 & .1471 \\ \mathrm{H} & 1.1004173 & 121.166909 & 1.552698 & .1469 \\ \mathrm{H} & 1.1040615 & 119.761470 & -179.376940 & .1693 \\ \mathrm{H} & 1.1218465 & 109.806496 & 160.010297 & .1142 \\ \mathrm{H} & 1.1226900 & 109.342531 & 39.463001 & .1045 \\ \mathrm{H} & 1.1216628 & 109.713347 & -79.793975 & .0853 \\ \mathrm{H} & 1.1008780 & 120.997246 & -177.548498 & .1468 \\ \mathrm{H} & 1.1033045 & 120.169015 & 178.440451 & .1689 \\ \mathrm{H} & 1.1044403 & 119.894897 & -.473129 & .1694 \\ \mathrm{H} & 1.1016472 & 117.782787 & -178.106232 & .1469 \\ \mathrm{H} & 1.1046121 & 120.011355 & 179.551147 & .1688 \\ \mathrm{H} & 1.1007447 & 120.983285 & -178.483778 & .1469 \\ \mathrm{H} & 1.1005477 & 120.894619 & 3.801425 & .1471 \\ \mathrm{H} & 1.1033446 & 119.908094 & -179.189534 & .1691 \\ \mathrm{H} & 1.1232775 & 109.482161 & 142.271957 & .1045 \\ \mathrm{H} & 1.1220044 & 109.785053 & 21.830882 & .1154 \\ \mathrm{H} & 1.1220821 & 109.845860 & -98.430452 & .0848\end{array}$

